\title{
DILATIONS SIMILAR TO A SELF-ADJOINT OPERATOR
}

\author{
B. NAGY
}

Abstract. It is shown that every bounded linear operator $T$ in a complex Hilbert space $H$ is the $(1,1)$-compression of an operator in $H \oplus H$ that is similar to a self-adjoint operator.

Mathematics subject classification (2010): 47A20, 47A30.

Keywords and phrases: Polar decomposition, resolution of the identity, completely bounded operator valued measure, equivalent scalar products.

\section{REFERENCES}

[1] N. Dunford and J. T. Schwartz, Linear Operators, Parts II and III, Wiley-Interscience, New York, 1963, 1971.

[2] D. W. Hadwin, Dilations and Hahn decompositions for linear maps, Can. J. Math. 33 (1981), 826839.

[3] V. I. Paulsen, Completely Bounded Maps and Operator Algebras, Cambridge Univ. Press, Cambridge, 2002.

[4] J. RÄTZ, Comparison of inner products, Aeq. Math. 57 (1999), 312-321.

[5] C.-Y. SuEN, Completely bounded linear extensions of operator-valued functions on ${ }^{*}$-semigroups, Proc. Amer. Math. Soc. 105 (1989), 330-334.

[6] G. WitTstock, Ein operatorwertiger Hahn-Banach Satz, J. Funct. Anal. 40 (1981), 127-150. 